\title{
Vocal pitch in the human brain
}

Cell 174, 21-31.e9 (2018)

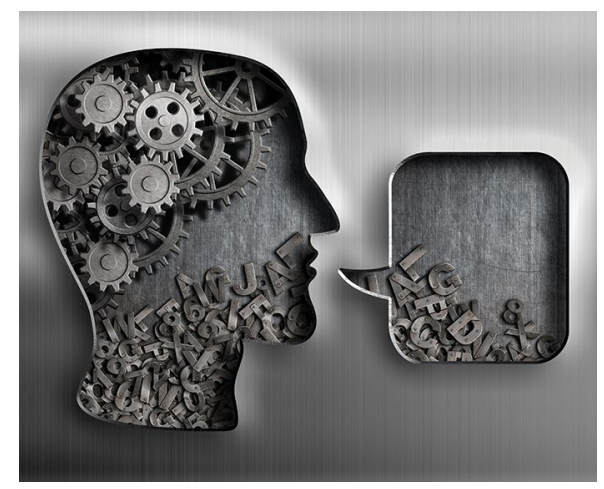

Credit: Andrey Kuzmin/Alamy Stock Photo

Human speech depends on precise control of the larynx, which is responsible both for making sounds audible and for creating the pitch at which they are produced. Though movements of the larynx have been correlated with activity in specific brain regions, it is not clear whether the brain is using similar mechanisms for general pitch control and pitch control in speech.

Benjamin Dichter, of University of California, San Francisco, and colleagues asked patients with implanted electrodes to read sentences, sometimes stressing specific words and sometimes reading naturally, and to sing a sequence of notes. The activity of the electrodes implanted in their brains showed that the region of their brains controlling the larynx - the dorsal laryngeal motor cortex - controlled pitch for both speaking and singing. To determine whether this activity was actually responsible for vocalization, the researchers stimulated the dorsal laryngeal motor cortex in awake patients undergoing craniotomies. This stimulation caused the patients to utter sounds. Taken together, these results identify the brain regions responsible for voluntary control of the larynx, the part of human anatomy that enables us to speak.

Mary Elizabeth Sutherland

Published online: 26 July 2018

https://doi.org/10.1038/s41562-018-0402-8 